\title{
Exposure to Perchlorate in Lactating Women and Its Associations With Newborn Thyroid Stimulating Hormone
}

\begin{abstract}
Yasemin Ucal ${ }^{1}$, Ozlem N. Sahin ${ }^{2}$, Muhittin Serdar ${ }^{1}$, Ben Blount ${ }^{3}$, Pinar Kumru, Murat Muhcu ${ }^{5}$, Mustafa Eroglu ${ }^{6}$, Cansu Akin-Levi ${ }^{1}$, Z. Zeynep Yildirim Keles ${ }^{1}$, Cem Turam ${ }^{1}$, Liza Valentin-Blasini ${ }^{3}$, Maria Morel-Espinosa ${ }^{3}$, Mustafa Serteser ${ }^{1}$, Ibrahim Unsal ${ }^{7}$ and Aysel Ozpinar ${ }^{1 *}$
\end{abstract}

${ }^{1}$ Department of Medical Biochemistry, School of Medicine, Acibadem Mehmet Ali Aydinlar University, Istanbul, Turkey, ${ }^{2}$ Department of Pediatrics, School of Medicine, Acibadem Mehmet Ali Aydinlar University, Istanbul, Turkey, ${ }^{3}$ Centers for Disease Control and Prevention, Atlanta, GA, United States, ${ }^{4}$ Department of Obstetrics and Gynecology, Zeynep Kamil Research and Training Hospital, Istanbul, Turkey, ${ }^{5}$ Department of Obstetrics and Gynecology, Haydarpasa Hospital of Gülhane Military Practice School and Hospital, Istanbul, Turkey, ${ }^{6}$ Department of Obstetrics and Gynecology, Haydarpasa Numune Training and Research Hospital, Istanbul, Turkey, ${ }^{7}$ Acibadem LabMed Laboratories, Istanbul, Turkey

\section{OPEN ACCESS}

Edited by:

Joanne F. Rovet,

Hospital for Sick Children, Canada

Reviewed by:

Angela Leung,

University of California, Los Angeles,

United States

Bijay Vaidya,

University of Exeter, United Kingdom

${ }^{*}$ Correspondence: Aysel Ozpinar

aysel.ozpinar@acibadem.edu.tr

Specialty section:

This article was submitted to

Thyroid Endocrinology,

a section of the journal

Frontiers in Endocrinology

Received: 10 March 2018

Accepted: 11 June 2018

Published: 03 July 2018

Citation:

Ucal Y, Sahin ON, Serdar M, Blount B,

Kumru P, Muhcu M, Eroglu M, Akin-Levi C, Keles ZZY, Turam C, Valentin-Blasini L, Morel-Espinosa M,

Serteser M, Unsal I and Ozpinar A (2018) Exposure to Perchlorate in Lactating Women and Its Associations

With Newborn Thyroid Stimulating

Hormone. Front. Endocrinol. 9:348.

doi: 10.3389/fendo.2018.00348
Background: Perchlorate, thiocyanate, and nitrate can block iodide transport at the sodium iodide symporter (NIS) and this can subsequently lead to decreased thyroid hormone production and hypothyroidism. NIS inhibitor exposure has been shown to reduce iodide uptake and thyroid hormone levels; therefore we hypothesized that maternal NIS inhibitor exposure will influence both maternal and newborn thyroid function.

Methods: Spot urine samples were collected from 185 lactating mothers and evaluated for perchlorate, thiocyanate, and nitrate concentrations. Blood and colostrum samples were collected from the same participants in the first $48 \mathrm{~h}$ after delivery. Thyroid hormones and thyroid-related antibodies (TSH, $\mathrm{TT} 3, \mathrm{TT} 4$, anti-TPO, anti-Tg) were analyzed in maternal blood and perchlorate was analyzed in colostrum. Also, spot blood samples were collected from newborns $(n=185)$ between 48 and 72 postpartum hours for TSH measurement. Correlation analysis was performed to assess the effect of NIS inhibitors on thyroid hormone levels of lactating mothers and their newborns in their first 48 postpartum hours.

Results: The medians of maternal urinary perchlorate (4.00 $\mu \mathrm{g} / \mathrm{g}$ creatinine), maternal urinary thiocyanate $(403 \mu \mathrm{g} / \mathrm{g}$ creatinine), and maternal urinary nitrate $(49,117 \mu \mathrm{g} / \mathrm{g}$ creatinine) were determined. Higher concentrations of all three urinary NIS inhibitors ( $\mu \mathrm{g} / \mathrm{g}$ creatinine) at their 75 th percentile levels were significantly correlated with newborn TSH $(r=0.21, p<0.001)$. Median colostrum perchlorate level concentration of all 185 participants was $2.30 \mu \mathrm{g} / \mathrm{L}$. Colostrum perchlorate was not significantly correlated with newborn TSH $(\rho>0.05)$; however, there was a significant correlation between colostrum perchlorate level and maternal TSH $(r=0.21, p<0.01)$. Similarly, there was a significant positive association between colostrum perchlorate and maternal urinary creatinine adjusted perchlorate $(r=0.32, p<0.001)$. 
Conclusion: NIS inhibitors are ubiquitous in lactating women in Turkey and are associated with increased TSH levels in newborns, thus signifying for the first time that co-exposure to maternal NIS inhibitors can have a negative effect on the newborn thyroid function.

Keywords: NIS inhibitors, perchlorate, nitrate, thiocyanate, colostrum, newborn thyroid health

\section{INTRODUCTION}

The thyroid hormones T4 and T3 are essential for normal development and survival of the human newborn. In infants, chronically low levels of thyroid hormones can lead to mental deficiency and motor deficits such as spasticity, dystonia or rigidity (1). TSH, which is secreted from the anterior pituitary gland, stimulates iodine trapping, thyroid hormone production, and thyroid hormone release from the thyroid gland. Thyroid hormone production requires adequate iodine intake, as iodine is a critical component of T4 and T3. The sodium iodide symporter (NIS) is unique in that it transports iodine into the thyroid gland of mother and fetus, as well as the newborn child. Additionally, NIS is also expressed at the lactating breast and mediates active transport of iodine into the milk for thyroid hormone synthesis by the newborn (2). Several environmentally-derived competitive NIS inhibitors, particularly perchlorate, thiocyanate, and nitrate, are known to inhibit both T4 and T3 production and lead to increased TSH production $(3,4)$. In the presence of environmentally-derived inhibitors, they can also be actively transported into the breast milk and subsequently be consumed by infants. Therefore, exposure of lactating women to perchlorate, thiocyanate, and nitrate may affect both maternal and newborn thyroid function and health.

Perchlorate is an inorganic ion that is widely manufactured for use in rocket propellant systems, matches, fireworks, and other industrial applications $(5,6)$. Perchlorate can also form naturally in the atmosphere and accumulate in the soil of arid regions (5). Moreover, perchlorate from both natural and manufactured sources can be concentrated in foods such as green vegetables, water, eggs, and milk (7). Human perchlorate exposure arises when perchlorate-contaminated food and drinking water are consumed (8). Thiocyanate is the primary metabolite of hydrogen cyanide in cigarette smoke and also can be formed after digestion of some plant-based foods (9). Nitrates derived from agricultural fertilizer runoff or exposure to sewage are commonly found in food and drinking water (10). Thiocyanate and nitrate less effectively inhibit iodide transport than perchlorate (11).

Thyroid health is an important global issue since mild iodine deficiency is prevalent in many parts of the world, including some European countries, the USA and Australia (12) and exposure to NIS inhibitors is ubiquitous (13-16). Additionally, Turkey has mild iodine deficiency (17) and a high intake of the iodide uptake inhibitor perchlorate is detected in Turkish populations (15). Because information on the effect of the NIS inhibitors on lactating women and newborns is limited in the literature, we proposed to study this in Turkish women and their newborns (18-20). To our knowledge, this is the first study to evaluate exposure to NIS inhibitors in lactating women and the association between perchlorate exposure and newborn TSH levels.

\section{MATERIALS AND METHODS}

\section{Recruitment of Subjects}

Between June 2013 and August 2013, 258 healthy pregnant women with a singleton pregnancy were recruited at routine examinations in week 38 at the state hospital specialized in women obstetrics and gynecology-Zeynep Kamil Research and Training Hospital-in Istanbul, Turkey. This state hospital was purposefully selected because it is one of the major obstetrics and gynecology hospitals in Turkey and provides a large samplesize of healthy pregnant women. An experienced doctor recruited healthy pregnant women in the clinic. Any woman with a past diagnosis of thyroid disorders, chronic illnesses, use of thyroidactive medication (e.g., amiodarone, glucocorticoids, dopamine, propranolol, iodine, lithium, phenytoin, carbamazepine) was excluded. Of the 258 eligible pregnant women, 200 met inclusion criteria and gave informed consent. Informed consent was obtained at the time of enrollment of the women in the study. Only women who could provide a sufficient amount of colostrum $(\sim 5 \mathrm{ml})$, were included in the study. Since sufficient colostrum to measure perchlorate was obtained for only 185 samples, 15 women were eliminated from the original sample.

All subjects resided in suburban Istanbul and provided standardized information on their medical histories and lifestyles, including cigarette smoke exposure, diabetes history, age, residency, and family medical history. This study was approved by Acibadem University Ethics Committee (ATADEK 2013-507).

\section{Sample Collection}

Samples were obtained between June and August 2013. In the first 48 postpartum hours, urine samples were collected from mothers using standard urine collection containers, as described previously (15), while blood samples were obtained in blood serum tubes. Colostrum samples were collected from the 185 lactating women as soon as lactation began, with the timing being within 72 postpartum hours for subjects having C-section and within 48 postpartum hours for subjects having spontaneous vaginal delivery. Colostrum samples were collected into sterile sample collection cups via manual expression. Additionally, newborn heel-prick blood was obtained between 48 and $72 \mathrm{~h}$ after delivery to measure newborn TSH levels. Colostrum, urine, 

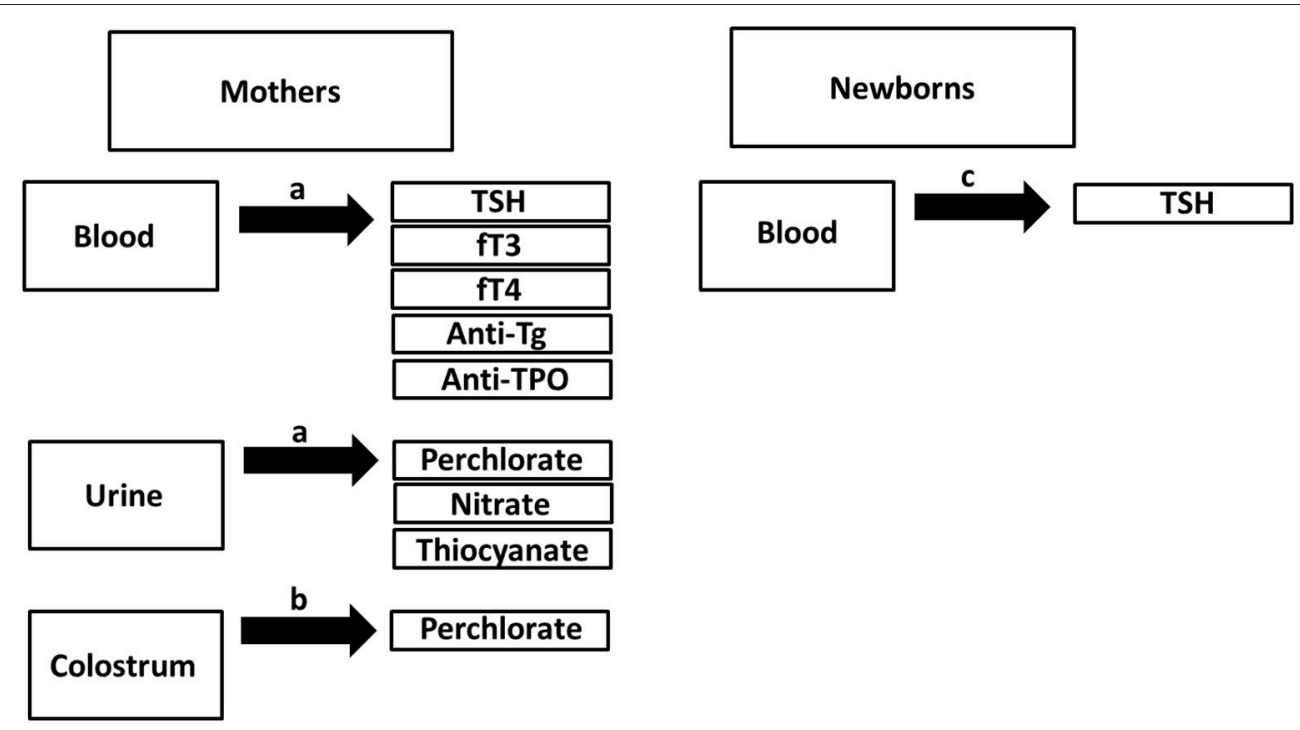

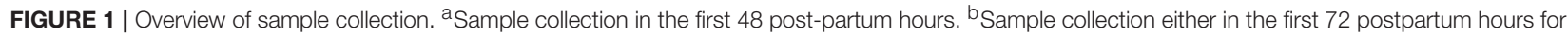
subjects having $\mathrm{C}$-section or 48 postpartum hours for subjects having vaginal delivery. ${ }^{\mathrm{C}}$ Spot blood collection using heel prick test $>48$ th postpartum hours.

serum, and newborn serum samples were kept at $-80^{\circ} \mathrm{C}$ until analysis. Sample collection procedures are detailed in Figure 1.

\section{Laboratory Measurements and Calculations}

Perchlorate, thiocyanate, and nitrate levels were measured in maternal urine samples while perchlorate was additionally measured in colostrum samples. Thiocyanate is unstable in colostrum, and therefore not measured (B. Blount, personal communication). Analyses of maternal urinary perchlorate, thiocyanate, nitrate, and colostrum perchlorate were performed on triple quadrupole ion chromatography-mass spectrometry at the Centers for Disease Control and Prevention. The method was modified from Valentin-Blasini et al. (21). A modified version of the Jaffe method was used for measuring urinary creatinine concentrations (Roche Diagnostics Corp., IN, USA) (22). Creatinine excretion rates were calculated using the method detailed in our previous publication (15). All reported data complied with rigorous quality control procedures of the CDC Division of Laboratory Sciences (23).

Maternal fT3, fT4, TSH, anti-thyroid peroxidase (TPO) antibodies, and anti-thyroglobulin (anti-Tg) antibodies from serum samples were determined by electro-chemiluminescence immunoassay (ECLIA) using Elecsys 2010 (Roche Diagnostics, Germany).

Newborn perchlorate intake was estimated based on the perchlorate concentrations in breast milk from the US Environmental Protection Agency (EPA) Exposure Handbook and reported rates of breast milk consumption by newborns as $100-120 \mathrm{ml} / \mathrm{kg} /$ day (24).

\section{Statistical Analysis}

We used descriptive statistics (mean, median, first and third quartiles, and standard deviation) to characterize subjects' demographic and clinical variables. Continuous values were represented as medians and interquartile ranges (IQR). ShapiroWilk test was applied to determine data normality. Subjects' data were examined by Pearson and Spearman correlations using Analyse-it Software (Analyse-it Software, Ltd. United Kingdom, V4.20.1). The relationship between subjects' demographic and clinical variables or between the two variables and NIS inhibitors were performed using correlation analysis. Bonferroni correction was applied to reduce type I errors in the multiple tests.

Maternal co-exposure to NIS inhibitors was assessed by establishing subgroups in the study population. For this purpose, subjects with higher than $75 \%$ maternal urinary NIS inhibitor concentration were selected and assigned to four groups: those having three NIS inhibitors elevated, those having two, those having just one, and those have none Statistical significance between four groups was determined by one-way analysis of variance (ANOVA) followed by Bonferroni's post-hoc test, considering $p \leq 0.05$ as significantly different.

\section{RESULTS}

\section{Demographic Characteristics and Thyroid Function Test Results}

The primary study participant characteristics [i.e., maternal age, body mass index (BMI), maternal TSH, maternal $\mathrm{fT} 3$, maternal fT4, maternal anti-TPO and anti-Tg levels] are summarized in Table 1. Newborn TSH levels, birth weights and estimated perchlorate intake levels are given in Table 2. FT4 was negatively correlated with the BMI of the 185 participants $(r=-0.20, p=$ $0.01)$. In addition, there was a negative trend between newborn TSH and maternal fT3. 
TABLE 1 | Maternal characteristics and maternal thyroid hormone function tests $(n=185)$.

\begin{tabular}{|c|c|c|c|c|c|}
\hline Variable & Mean & $S D$ & 25th percentile & Median & 75th percentile \\
\hline Age & 27.08 & 5.63 & 23.00 & 27.00 & 30.00 \\
\hline $\mathrm{BMI}^{\phi}\left(\mathrm{kg} / \mathrm{m}^{2}\right)$ & 29.10 & 4.50 & 25.80 & 28.70 & 32.00 \\
\hline TSH ( $\mu \mathrm{U} / \mathrm{ml})$ & 2.56 & 1.94 & 1.40 & 2.20 & 3.10 \\
\hline fT3 (pmol/L) & 4.52 & 0.75 & 4.00 & 4.30 & 4.80 \\
\hline fT4 (pmol/L) & 12.06 & 2.04 & 10.70 & 12.00 & 13.20 \\
\hline AntiTPO (IU/ml) & 13.98 & 35.88 & 5.00 & 6.00 & 8.40 \\
\hline Anti TG (IU/ml) & 30.19 & 85.50 & 12.20 & 16.90 & 21.40 \\
\hline
\end{tabular}

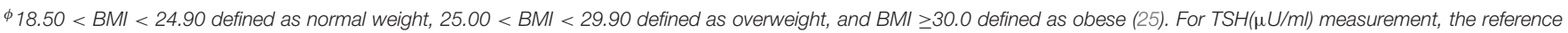

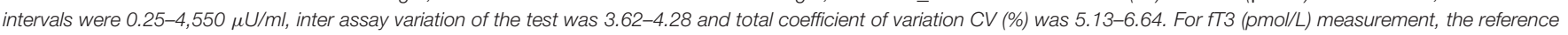

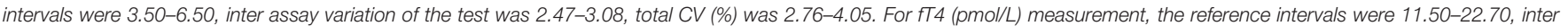
assay variation of the test was $2.33-4.00$, total $\mathrm{CV}(\%)$ was $3.44-4.58$.

TABLE 2 | Newborn characteristics, TSH concentrations and perchlorate intake estimations $(n=185)$.

\begin{tabular}{|c|c|c|c|c|c|}
\hline Variable & Mean & $S D$ & 25th Percentile & Median & 75th Percentile \\
\hline Birth weight (g) & 3,204 & 502 & 2,960 & 3,160 & 3,520 \\
\hline $\mathrm{TSH}(\mu \mathrm{U} / \mathrm{ml})^{\phi}$ & 4.62 & 3.26 & 2.50 & 3.80 & 6.10 \\
\hline Perchlorate intake estimation $¥$ ( $\mu \mathrm{g} / \mathrm{kg} /$ day) & 0.20 & 0.23 & 0.00 & 0.10 & 0.30 \\
\hline
\end{tabular}

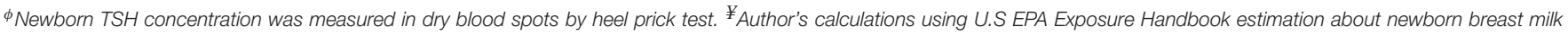
consumption rate of $100-120 \mathrm{ml} / \mathrm{kg} /$ day (24).

\section{Association Between Maternal Urinary NIS Inhibitors and Maternal Thyroid Function Test Results}

The median maternal urinary perchlorate $(4.00 \mu \mathrm{g} / \mathrm{g}$ creatinine), maternal urinary thiocyanate (403 $\mu \mathrm{g} / \mathrm{g}$ creatinine) and maternal urinary nitrate $(49,117 \mu \mathrm{g} / \mathrm{g}$ creatinine $)$ were determined (Table 3). Urinary nitrate was strongly correlated with both thiocyanate $(r=0.36, p<0.0001)$ and perchlorate $(r=0.44$; $p<$ 0.0001).

For the 185 women, there was no correlation between maternal TSH and any creatinine-adjusted NIS inhibitor levels. Additionally, there was no statistically significant difference between co-exposure to maternal NIS inhibitors and maternal TSH.

There were no significant correlations between free thyroid hormones (fT3 and fT4) and any maternal urinary NIS inhibitors nor between thyroid auto-antibodies (anti-TPO and anti-Tg) and any NIS inhibitors.

\section{Association Between Maternal Urinary NIS Inhibitors and Newborn TSH}

For the 185 newborns, a significant correlation was seen between newborn-screen TSH and maternal urinary perchlorate $(r=$ $0.24, p<0.001$ ) but not with maternal urinary thiocyanate and nitrate concentrations. However, there was no significant correlation between newborn TSH and any creatinine-adjusted maternal urinary NIS inhibitors. Nevertheless, when newborn
TSH and 75th percentile creatinine-adjusted urinary perchlorate, thiocyanate and nitrate levels of lactating women were compared in a regression analysis model, women with the highest quartile urinary concentrations of all 3 NIS inhibitors had newborns with higher newborn TSH levels $(r=0.21, p<0.001)$ (Figure 2).

\section{Maternal Perchlorate Concentration in Colostrum and Its Association With Maternal and Newborn TSH}

The median maternal perchlorate concentration in colostrum was $2.30 \mu \mathrm{g} / \mathrm{L}$. Calculation of estimated newborn perchlorate intake (24) revealed a median dose of $0.10 \mu \mathrm{g} / \mathrm{kg} / \mathrm{day}$. Colostrum perchlorate concentration was significantly correlated with maternal urinary creatinine-adjusted perchlorate $(r=0.32, p<$ $0.001)$ and with maternal TSH $(r=0.21, p<0.01)$ (Figure 3), but not with newborn TSH.

\section{DISCUSSION}

The present study represents the first assessment of NIS inhibitor exposure in lactating women and their newborns in Turkish populations. Results from this study showed that NIS inhibitors were ubiquitous in lactating women. Additionally, lactating women with the highest quartile urinary concentrations of all 3 NIS inhibitors had newborns with higher newborn TSH levels. Previous studies which have evaluated the relationship of any effects of NIS inhibitors on newborns and/or infants 
TABLE 3 | Maternal urinary NIS inhibitor concentrations and maternal colostrum perchlorate concentration $(n=185)$.

\begin{tabular}{|c|c|c|c|c|c|}
\hline Variable & Mean & $S D$ & 25th percentile & Median & 75th percentile \\
\hline Urinary perchlorate ( $\mu \mathrm{g} / \mathrm{g}$ creatinine) & 5.60 & 5.69 & 2.30 & 4.00 & 6.30 \\
\hline Urinary thiocyanate ( $\mu \mathrm{g} / \mathrm{g}$ creatinine) & 610 & 802 & 207 & 403 & 686 \\
\hline Urinary nitrate ( $\mu \mathrm{g} / \mathrm{g}$ creatinine) & 62,289 & 62,901 & 34,228 & 49,117 & 67,881 \\
\hline Colostrum perchlorate ( $\mu \mathrm{g} / \mathrm{L})$ & 4.09 & 4.75 & 1.20 & 2.30 & 5.70 \\
\hline
\end{tabular}

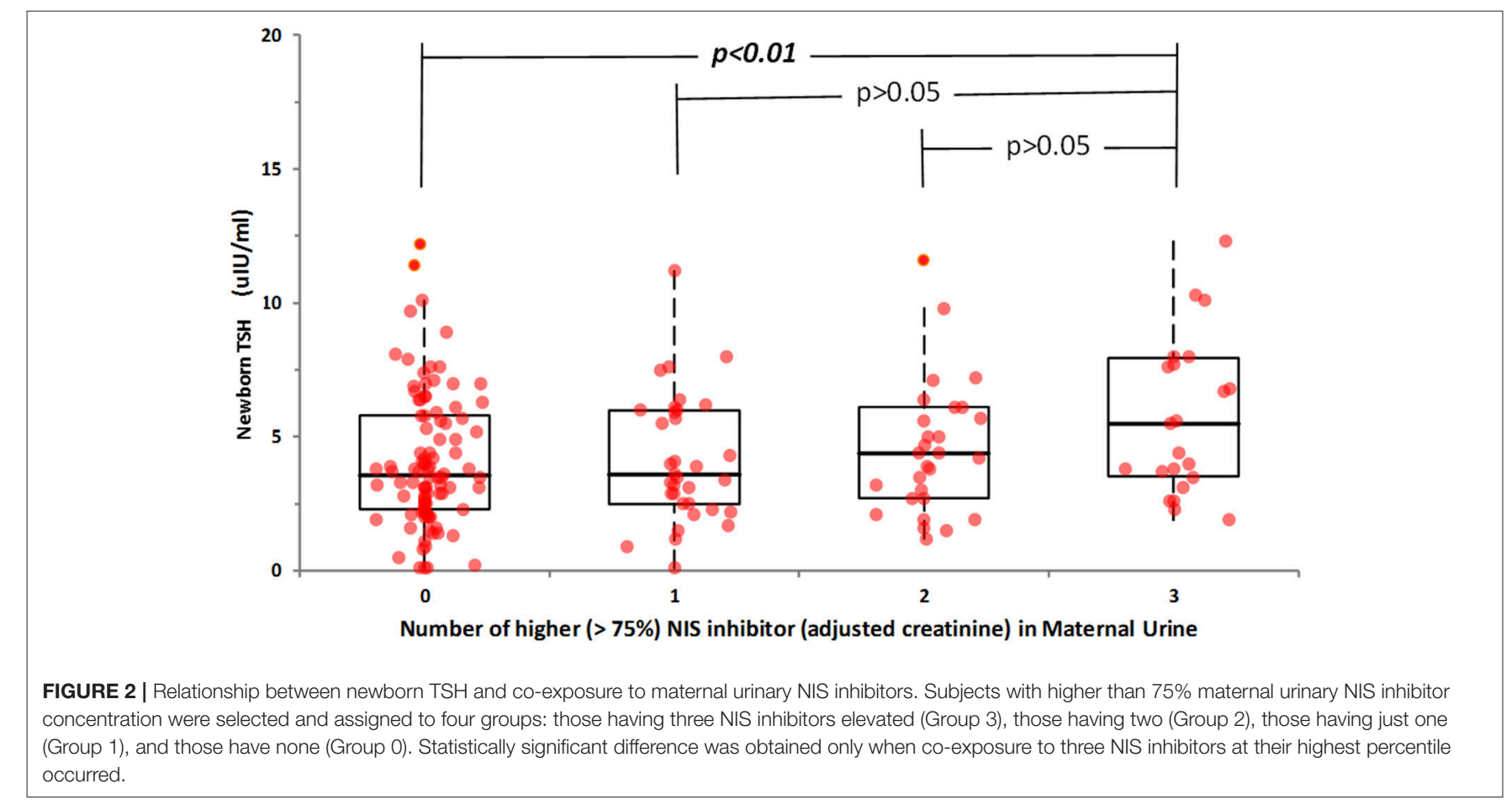

showed no associations between environmental perchlorate exposure and newborn and/or infant thyroid function (21, 22, 25), with one exception. The latter was a study reporting higher newborn $\mathrm{SH}$ with high levels of perchlorate in drinking water during pregnancy (20). To the best of our knowledge, the present study is the first to assess the effect of potential co-exposure to all three NIS inhibitors on newborn thyroid function. Thus, our results suggest that co-exposure to maternal NIS inhibitors can negatively affect newborn thyroid health.

We found that median urinary perchlorate concentration in Turkish lactating women $(3.89 \mu \mathrm{g} / \mathrm{L})$ was relatively higher than in the U.S. lactating women $[3.0 \mu \mathrm{g} / \mathrm{L}(26), 3.1 \mu \mathrm{g} / \mathrm{L}(27)]$. This result is consistent with our previously published work, in which we evaluated urinary perchlorate concentrations in non-pregnant and non-lactating women and found that median urinary perchlorate concentration $(6.4 \mu \mathrm{g} / \mathrm{L})$ was more than twice as high as the median concentration found in U.S. women $(2.9 \mu \mathrm{g} / \mathrm{L})(15,16)$. We found similar median concentrations for maternal urinary thiocyanate and nitrate in this study and in our previously published work including non-lactating and non-pregnant women (15). Interestingly, the median maternal urinary thiocyanate concentrations $(274 \mu \mathrm{g} / \mathrm{L})$ in our study were relatively lower than the U.S. lactating women $(514 \mu \mathrm{g} / \mathrm{L})(27)$. We believe this discrepancy is not related to the number of active smokers in the study groups since only a few women actively smoked in both groups during lactation. One possible explanation for this discrepancy is lactating Turkish women smoke with less frequency and intensity compared with U.S. lactating women.

The finding that no significant correlations between maternal TSH and any maternal urinary NIS inhibitors are congruent with studies reporting lack of association between elevated TSH levels and NIS inhibitor exposure $(16,22,28)$. However, a crosssectional study of the US population showed that increased urinary perchlorate was significantly associated with increased TSH levels (29). Possible explanations may account for the discrepancy in the literature are the population demographics and characteristics-e.g., pregnant vs. non-pregnant-genetic differences in NIS metabolism and differences in methodologies. 


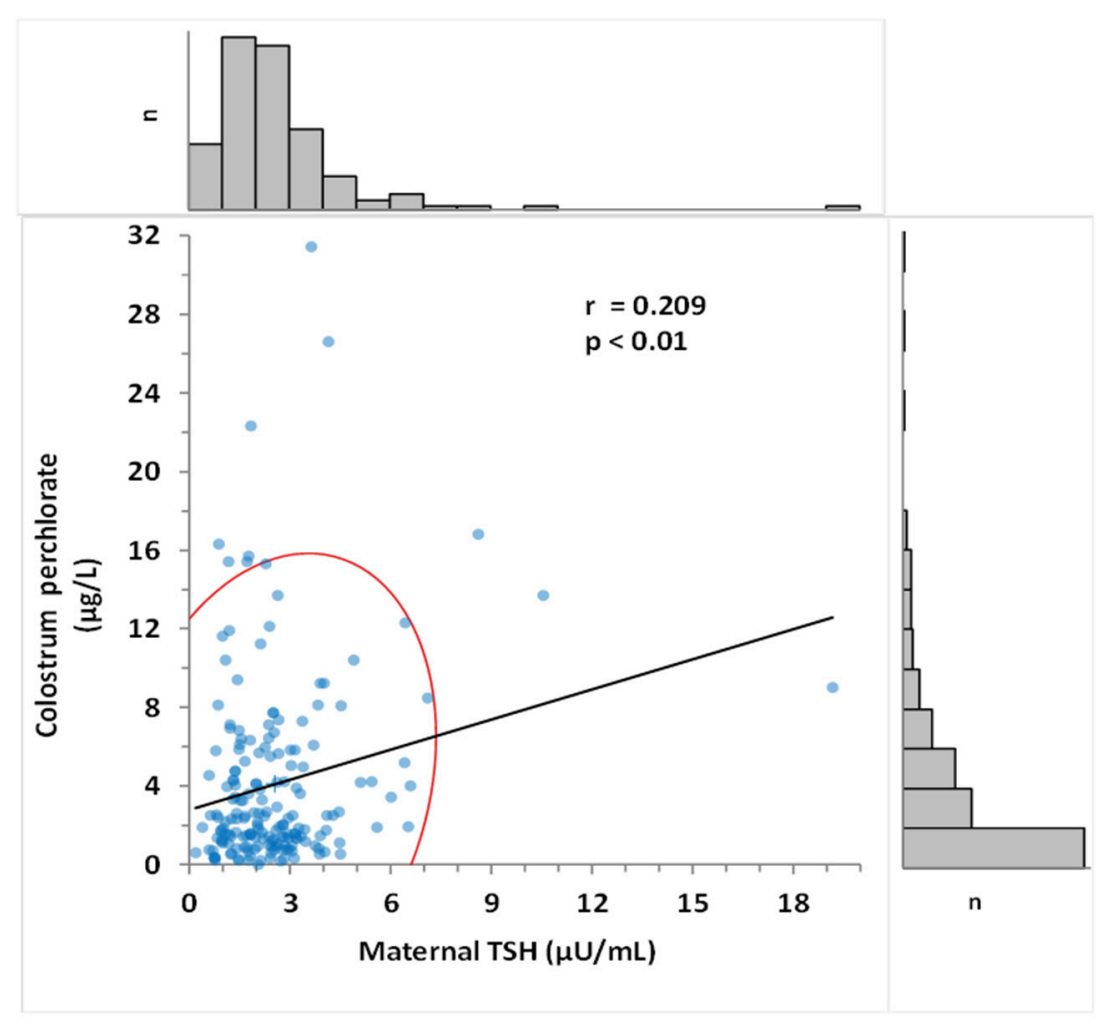

FIGURE 3 | Correlation between colostrum perchlorate levels and maternal TSH. Pearson's correlation coefficient was determined as 0.209 ( $p<0.015)$. Frequency histogram shows the number of values ( $\mathrm{n}$ ) in the corresponding axis. The red curve represents $95 \% \mathrm{Cl}$ of the distribution.

The median colostrum perchlorate concentration in our study $(2.30 \mu \mathrm{g} / \mathrm{L})$ was remarkably similar to the previously published perchlorate levels found in colostrum collected from lactating U.S. women (30). Our findings were consistent with the median perchlorate levels reported for breast milk by Kirk et al., but lower than the mean perchlorate concentration $(10.5 \mu \mathrm{g} / \mathrm{L})$, indicative of the right-skewed distribution of perchlorate in the Kirk et al. study (28). Different study participants across different studies may have had different perchlorate exposures from differences in perchlorate intake from diet and drinking water. Alternatively, perchlorate secretion into breast milk could change with time: hormonal changes in lactation could affect NIS expression and thus result in differential NIS inhibitor secretion into colostrum vs. breast milk. For example, prolactin mediates iodine accumulation in cultured mammary tissues (31).

The perchlorate intake estimation in newborns revealed that their intake was below the U.S. EPA reference dose of $0.70 \mu \mathrm{g} / \mathrm{kg} /$ day (32) and similar to the median perchlorate intake $(0.16 \mu \mathrm{g} / \mathrm{kg} /$ day $)$ estimated by Valentin-Blasini et al. in U.S. infants (24). We currently found no significant correlation between colostrum perchlorate concentrations and newborn TSH. The reason for this non-significant effect could be that in the first $48 \mathrm{~h}$ postpartum may be too early to adequately determine the relationship between colostrum perchlorate concentration and newborn thyroid health status. On the other hand, these levels may be unrelated given Leung et al.'s finding that breast milk perchlorate concentration and infant TSH were unrelated (19).

Although we found no significant relationship between colostrum perchlorate concentrations and newborn TSH, a significant positive correlation was observed between colostrum perchlorate concentrations and maternal TSH levels. Importantly, this finding may provide important evidence that environmental perchlorate exposure is associated with increased production of maternal TSH and, at higher quartiles of co-exposure, leads to increased newborn TSH.

This study provides novel data indicating that lactating study participants have a high intake of some NIS inhibitors and that this may affect newborn thyroid health. However, our study is limited in that maternal urinary iodine concentrations substantially varied between study participants (data not shown), which may be related to usage of iodine-containing disinfectant for skin preparation before delivery. Since we believed different cutaneous absorption of available iodine could have resulted in variations, urinary iodine concentrations were not included in the analysis.

\section{CONCLUSION}

The present study provides the first indication that co-exposure to three common maternal NIS inhibitors, namely perchlorate, thiocyanate, and nitrate may alter newborn TSH levels. We 
found that increased newborn TSH levels were associated with higher quartiles of co-exposure to the maternal NIS inhibitors. Our results therefore signify an important first step in the investigation of co-exposure to NIS inhibitors on newborn thyroid function. However, given that colostrum perchlorate concentration appeared to have no effect on newborn thyroid function, further study is warranted. Overall, this study provides critical new insights into the effects of co-exposure to maternal NIS inhibitors on newborn thyroid function. In addition, the results given here have important implications for the evaluation of NIS inhibitor exposure in lactating women and its potential effect on newborn thyroid health status in Turkish populations.

\section{REFERENCES}

1. Agrawal P, Philip R, Saran S, Gutch M, Razi MS, Agroiya P, et al. Congenital hypothyroidism. Indian J Endocrinol Metab. (2015) 19:221-7. doi: $10.4103 / 2230-8210.131748$

2. Dohan O, De la Vieja A, Paroder V, Riedel C, Artani M, Reed M, et al. The sodium/iodide Symporter (NIS): characterization, regulation, and medical significance. Endocr Rev. (2003) 24:48-77. doi: 10.1210/er.20010029

3. Kogai T, Brent GA. The sodium iodide symporter (NIS): regulation and approaches to targeting for cancer therapeutics. Pharmacol Ther. (2012) 135:355-70. doi: 10.1016/j.pharmthera.2012.06.007

4. Leung AM, Pearce EN, Braverman LE. Perchlorate, iodine and the thyroid. Best Pract Res Clin Endocrinol Metab. (2010) 24:133-41. doi: 10.1016/j.beem.2009.08.009

5. Dasgupta PK, Martinelango PK, Jackson WA, Anderson TA, Tian K, Tock RW, et al. The origin of naturally occurring perchlorate: the role of atmospheric processes. Environ Sci Technol. (2005) 39:1569-75. doi: $10.1021 /$ es048612x

6. Erickson BE. Tracing the origin of perchlorate. A main ingredient in rocket fuel is showing up almost everywhere researchers look, but where is it all coming from? Anal Chem. (2004) 76:388A-9. doi: 10.1021/ac0416620

7. Blount BC, Ozpinar A, Alwis KU, Caudill SP, Gillespie JR. Perchlorate, nitrate, thiocyanate, and iodide levels in chicken feed, water, and eggs from three farms. J Agric Food Chem. (2008) 56:10709-15. doi: 10.1021/jf80 18326

8. Can O, Blount B, Valentin-Blasini L, Erdemgil Y, Uzunoglu D, Aksoy $\mathrm{M}$, et al. Perchlorate exposure through water and milk in Istanbul. Bull Environ Contam Toxicol. (2016) 97:439-45. doi: 10.1007/s00128-0161889-1

9. Chandler JD, Day BJ. Thiocyanate: a potentially useful therapeutic agent with host defense and antioxidant properties. Biochem Pharmacol. (2012) 84:1381-7. doi: 10.1016/j.bcp.2012.07.029

10. Hord NG, Tang Y, Bryan NS. Food sources of nitrates and nitrites: the physiologic context for potential health benefits. Am J Clin Nutr. (2009) 90:1-10. doi: 10.3945/ajen.2008.27131

11. Tonacchera M, Pinchera A, Dimida A, Ferrarini E, Agretti P, Vitti P, et al. Relative potencies and additivity of perchlorate, thiocyanate, nitrate, and iodide on the inhibition of radioactive iodide uptake by the human sodium iodide symporter. Thyroid (2004) 14:1012-9. doi: 10.1089/thy.2004.14.1012

12. Richard K, Li H, Landers KA, Patel J, Mortimer RH. Placental transport of thyroid hormone and iodide. In: J. Zheng editor. Recent Advances in Research on the Human Placenta. Rijeka: InTech. (2012). p. 309-34.

13. Steinmaus C, Pearl M, Kharrazi M, Blount BC, Miller MD, Pearce EN, et al. Thyroid hormones and moderate exposure to perchlorate during pregnancy in women in Southern California. Environ Health Perspect. (2016) 124:861-7. doi: 10.1289/ehp.1409614

14. Mortensen ME, Birch R, Wong L-Y, Valentin-Blasini L, Boyle EB, Caldwell $\mathrm{KL}$, et al. Thyroid antagonists and thyroid indicators in U.S. pregnant women

\section{AUTHOR CONTRIBUTIONS}

AO was study PI and involved in all aspects of design, interpretation, and writing. BB led lab analysis and contributed to writing. YU wrote the manuscript with support from AO and BB. MusS, IU, and MuhS helped supervise the project. MuhS and OS contributed to the statistical analysis. LV-B and MM-E involved in lab analysis. PK, MM, and ME were involved in subject selection and coordinated sample collection at the hospital. CA-L, $\mathrm{ZK}$, and CT contributed to sample collection at the hospital. All authors provided critical feedback and helped shape the research, analysis and manuscript. in the Vanguard Study of the National Children's Study. Environ Res. (2016) 149:179-88. doi: 10.1016/j.envres.2016.05.017

15. Ozpinar A, Kelestimur F, Songur Y, Can O, Valentin L, Caldwell K, et al. Iodine status in Turkish populations and exposure to iodide uptake inhibitors. PLoS ONE (2014) 9:e88206. doi: 10.1371/journal.pone.008 8206

16. Blount BC, Valentin-Blasini L, Osterloh JD, Mauldin JP, Pirkle JL. Perchlorate exposure of the US Population, 2001-2002. J Expo Sci Environ Epidemiol. (2007) 17:400-7. doi: 10.1038/sj.jes.7500535

17. Andersson M, Karumbunathan V, Zimmermann MB. Global iodine status in 2011 and trends over the past decade. J Nutr. (2012) 142:744-50. doi: 10.3945/jn. 111.149393

18. Steinmaus C, Miller MD, Smith AH. Perchlorate in drinking water during pregnancy and neonatal thyroid hormone levels in California. J Occup Environ Med. (2010) 52:1217-24. doi: 10.1097/JOM.0b013e3181 fd6fa7

19. Leung AM, Braverman LE, He X, Schuller KE, Roussilhes A, Jahreis KA, et al. Environmental perchlorate and thiocyanate exposures and infant serum thyroid function. Thyroid (2012) 22:938-43. doi: 10.1089/thy.2012.0058

20. Tellez Tellez R, Michaud Chacon P, Reyes Abarca C, Blount BC, Van Landingham CB, Crump KS, et al. Long-term environmental exposure to perchlorate through drinking water and thyroid function during pregnancy and the neonatal period. Thyroid (2005) 15:963-75. doi: 10.1089/thy.2005.15.963

21. Valentin-Blasini L, Blount BC, Delinsky A. Quantification of iodide and sodium-iodide symporter inhibitors in human urine using ion chromatography tandem mass spectrometry. J Chromatogr A (2007) 1155:406. doi: 10.1016/j.chroma.2007.04.014

22. Junge W, Wilke B, Halabi A, Klein G. Determination of reference intervals for serum creatinine, creatinine excretion and creatinine clearance with an enzymatic and a modified Jaffe method. Clin Chim Acta (2004) 344:137-148. doi: 10.1016/j.cccn.2004.02.007

23. Yu LL, Jarrett JM, Davis WC, Kilpatrick EL, Oflaz R, Turk GC, et al. Characterization of perchlorate in a new frozen human urine standard reference material. Anal Bioanal Chem. (2012) 404:1877-86. doi: 10.1007/s00216-012-6263-5

24. Valentín-Blasini L, Blount BC, Otero-Santos S, Cao Y, Bernbaum JC, Rogan WJ. Perchlorate exposure and dose estimates in infants. Environ Sci Technol. (2011) 45:4127-32. doi: 10.1021/es103160j

25. Centers for Disease Control and Prevention. Body Mass Index (BMI). (2017). Available online at: https://www.cdc.gov/healthyweight/assessing/bmi/index.html

26. Pearce EN, Leung AM, Blount BC, Bazrafshan HR, He X, Pino S, et al. Breast milk iodine and perchlorate concentrations in lactating Boston-area women. $J$ Clin Endocrinol Metab. (2007) 92:1673-7. doi: 10.1210/jc.2006-2738

27. Lee SY, McCarthy AM, Stohl H, Ibrahim S, Jeong C, Braverman LE, et al. Urinary iodine, perchlorate, and thiocyanate concentrations in U.S. lactating women. Thyroid (2017) 27:1574-81. doi: 10.1089/thy.2017. 0158 
28. Kirk AB, Martinelango PK, Tian K, Dutta A, Smith EE, Dasgupta PK. Perchlorate and iodide in dairy and breast milk. Environ Sci Technol. (2005) 39:2011-17. doi: 10.1021/es048118t

29. Horton MK, Blount BC, Valentin-Blasini L, Wapner R, Whyatt R, Gennings $\mathrm{C}$, et al. Co-occurring exposure to perchlorate, nitrate and thiocyanate alters thyroid function in healthy pregnant women. Environ Res. (2015) 143:1-9. doi: 10.1016/j.envres.2015.09.013

30. Leung AM, Pearce EN, Hamilton T, He X, Pino S, Merewood A, et al. Colostrum iodine and perchlorate concentrations in Boston-area women: a cross-sectional study. Clin Endocrinol. (2009) 70:326-30. doi: 10.1111/j.1365-2265.2008.03330.x

31. Rillema JA, Yu TX, Jhiang SM. Effect of prolactin on sodium iodide symporter expression in mouse mammary gland explants. Am J Physiol Endocrinol Metab. (2000) 279:E769-72. doi: 10.1152/ajpendo.2000.279.4. E769
32. U.S. Environmental Protection Agency. Perchlorate and Perchlorate Salts. Available online at: https://www.epa.gov/iris.

Conflict of Interest Statement: The authors declare that the research was conducted in the absence of any commercial or financial relationships that could be construed as a potential conflict of interest.

Copyright (C) 2018 Ucal, Sahin, Serdar, Blount, Kumru, Muhcu, Eroglu, Akin-Levi, Keles, Turam, Valentin-Blasini, Morel-Espinosa, Serteser, Unsal and Ozpinar. This is an open-access article distributed under the terms of the Creative Commons Attribution License (CC BY). The use, distribution or reproduction in other forums is permitted, provided the original author(s) and the copyright owner(s) are credited and that the original publication in this journal is cited, in accordance with accepted academic practice. No use, distribution or reproduction is permitted which does not comply with these terms. 